\title{
Análisis FT-IR y DLS de una probable ruta química para la síntesis de nanopartículas de $\mathrm{CuInSe}_{2}$ : influencia de la concentración de Selenio
}

\author{
A. Suárez-Gómez ${ }^{\mathrm{a}}$, R. Castañeda-Valderrama ${ }^{\mathrm{a} *}$, M.A. Carreón-Álvarez ${ }^{\mathrm{a}}$ y. Manchez-Tizapa ${ }^{\mathrm{a}}$ \\ ${ }^{a} U d G$-CUVALLES, Carr. Guadalaj ara-Ameca km. 45.5, Ameca, Jalisco, México 46600 \\ *E-mail: rcvalderrama@profesores.valles.udg.mx
}

Recibido 31 octubre 2013, Aceptado 21 diciembre 2013

\section{Resumen}

En este trabajo se formula un probable esquema de reacción para la síntesis de nanopartículas de $\mathrm{CuInSe}_{2}$ basados en análisis FT-IR de los precursores y de las soluciones finales. Se tomará en cuenta la influencia de la concentración de Selenio en el material precursor y la influencia que esto pueda tener en el tamaño medio de partícula de la dispersión coloidal resultante usando, en nuestro caso, al Hexano como medio dispersante.

Palabras clave: CIS, nanopartículas, DLS, FT-IR, tinta semiconductora

\section{Introducción}

Las celdas solares basadas en calcogenuros y aleaciones ternarias de cobre emergieron hace casi tres décadas como una solución muy promisoria al problema de la reducción de costos en la fabricación de celdas solares. Así, usando esta familia de materiales, ha llegado a obtenerse más de $21 \%$ de efíciencia de conversión de energía solar a nivel de laboratorio 1 a la vez que se disminuyen los costos de producción frente a las obleas tradicionales de silicio 2. Por otra parte, los dispositivos fotovoltaicos basados en películas delgadas de estos materiales han mostrado ser intrínsecamente estables 3 lo cual ayuda a evadir la conocida desventaja de degradación típica de las celdas solares convencionales.

En particular, el compuesto ternario Diselenuro de Indio y Cobre (CuInSe $\mathrm{S}_{2}$ o CIS) es uno de los candidatos más factibles para aplicaciones fotovoltaicas basadas en películas delgadas debido fundamentalmente a propiedades estructurales y eléctricas bastante peculiares relacionadas en esencia a mecanismos intrínsecos de compensación de defectos 4-6.

Gracias a los avances en la síntesis de nanocristales semiconductores 7-9 ha podido reportarse la obtención de nanopartículas de CIS y materiales similares por diversos métodos químicos entre los cuales sobresalen los métodos solvotérmicos, la descomposición térmica de precursores y la síntesis química asistida por microondas. Más allá de las ventajas asociadas a los costos de fabricación, a estas bajas escalas de trabajo las nanopartículas muestran una banda prohibida más ancha (efecto de corrimiento al azul) que sus homólogos de bulto y una fuerte dependencia de sus propiedades eléctricas con la concentración de defectos intrínsecos. Esto último resalta el importante papel del control estructural y composicional durante la síntesis de este compuesto ternario.

Para reducir los costos y la complejidad asociados al proceso de obtención de celdas solares, se hace necesaria una lógica modificación en los métodos de síntesis que permitan obtener películas suficientemente delgadas y con la mayor área posible con un mínimo de tiempo de síntesis. Este dilema "costo-beneficio" ha sido atacado, para el caso de las películas delgadas fotovoltaicas de materiales basados en $\mathrm{CuInSe}_{2}$, mediante la selenización (reacción de algún compuesto gaseoso de Selenio (por ejemplo, $\mathrm{H}_{2} \mathrm{Se}$ ) con los óxidos intermedios de $\mathrm{Cu}$ e In a altas temperaturas sin necesidad de trabajar a alto vacío). Gracias a este procedimiento, relativamente bien estudiado y discutido en el caso de capas activas basadas en películas, se han obtenido materiales de aceptable calidad fotovoltaica y con buen control estructural y morfológico 10.

A pesar de esto, aún la relación costo-beneficio no es óptima para una explotación comercial a mayor escala y, ante ese escenario tecnológico, se hace necesario explorar el paso de la síntesis de películas delgadas nanoestructuradas a la síntesis de nanopartículas. Dichas nanopartículas, en el medio dispersante adecuado, pueden servir para ser depositadas como "tinta" sobre un substrato dado y así aumentar la eficiencia de conversión por unidad de área lo cual podría implicar un mejoramiento de las celdas solares basadas en $\mathrm{CuInSe} \mathrm{S}_{2}$ en lo que a relación costo-beneficio se refiere.

Esta aproximación al trabajo con materiales basados en $\mathrm{CuInSe}_{2}$ es relativamente novedosa 11,12 y la selenización a estas escalas de trabajo aún no es un proceso/fenómeno entendido del todo 13. A modo de ilustración, en este caso no se trataría con la reacción de gases de Selenio a altas temperaturas sino de la adición del elemento Selenio a temperaturas moderadas bajo ciertas condiciones experimentales.

Más aún, el régimen de selenización puede afectar la morfología de las nanopartículas así como la relación Área/Volumen de las mismas lo cual afecta las posibles eficiencias de conversión de un dispositivo de celda solar basado en este material. De acuerdo a trabajos relativamente recientes, 13-16, cuando se desean obtener nanopartículas de CIS en forma de dispersión coloidal, se debe poner especial atención a la incorporación del Selenio (Selenización) durante la síntesis. Por otra parte, es esperado que la calidad estructural y morfológica de dicho material dependa de diversas variables experimentales $(\mathrm{pH}$, Potencial Zeta, envejecimiento, concentraciones, tamaño hidrodinámico de la partícula, espesor de la doble capa de Stern, etc.) que son regidos a partir de la síntesis química de los mismos y con clara repercusión en el entorno iónico de las nanopartículas resultantes en el seno de la dispersión coloidal.

En el presente trabajo se presenta la síntesis química 
de nanopartículas de $\mathrm{CuInSe}_{2}$, caracterizada mediante la espectroscopía infrarroja (FT-IR), a la vez que se discute, gracias a dichas mediciones, el esquema de reacción más probable. Por otra parte, gracias a la medición del tamaño medio de partícula, se puede apreciar la influencia de la concentración del medio dispersante y del selenio sobre dicho parámetro.

\section{Parte experimental}

Los reactivos de partida fueron Cloruro de cobre (I) (CuCl, Aldrich 229628, $\geq 99.995 \%)$, Cloruro de indio (III) $\left(\mathrm{InCl}_{3}\right.$, Aldrich 203440, 99.999\%), Selenio (Se, Aldrich 229865, 99.99\%), Oleilamina (OA, Aldrich O7805, 70\%), Hexano (Hex, Aldrich 296090, 95\%) y Etanol (EtOH, Aldrich 459844, $\geq 99.5 \%$ ).

Inicialmente, y por separado, se prepararon soluciones de $\mathrm{CuCl}$ y de $\mathrm{InCl}_{3}$ en $\mathrm{OA}$ a $0.2 \mathrm{M}$ mezclando las cantidades estequiométricas apropiadas de cada reactivo en un matraz y calentándolas con agitación hasta $130{ }^{\circ} \mathrm{C}$ por 1 hora. Estas soluciones serán designadas por $\mathrm{CuCl} / \mathrm{OA}$ e $\mathrm{InCl}_{3} / \mathrm{OA}$, respectivamente. En el caso del selenio, las soluciones en OA fueron preparadas a $0.25 \mathrm{M}$ y a $0.40 \mathrm{M}$ mezclando las cantidades estequiométricas apropiadas en un matraz y calentándolas con agitación hasta $180{ }^{\circ} \mathrm{C}$ por 2 horas. A estas soluciones les llamaremos Se/OA25 y Se/OA40, respectivamente.

En un paso posterior se realizó la síntesis de CIS usando los diferentes precursores de Selenio (Se/OAx; $\mathrm{x}$ $=25,40)$. Designaremos a cada material sintetizado como CIS25 y CIS40 según el tipo de precursor Selenio utilizado. Dicha síntesis se realizó como se describe a continuación.

En un matraz de 3 bocas calentamos $3 \mathrm{ml}$ de OA por encima de $120{ }^{\circ} \mathrm{C}$ durante media hora y añadimos, según la estequiometría prevista, los volúmenes correspondientes de $\mathrm{CuCl} / \mathrm{OA}, \mathrm{InCl}_{3} / \mathrm{OA}$ y $\mathrm{Se} / \mathrm{OAx}$, en ese orden. El sistema fue calentado lentamente hasta 250 ${ }^{\circ} \mathrm{C}$ y dicha temperatura se mantuvo durante 2 horas. Acto seguido, el sistema se dejó enfriar lentamente hasta los $50-60{ }^{\circ} \mathrm{C}$ aproximadamente y entonces se extrajeron unos pocos mililitros de material que fueron dispersados en Hexano (Hex) con proporciones volumétricas de $1: 150,1: 300$ y $1: 500$.

\subsection{Caracterizaciones experimentales}

Tanto las soluciones de partida como las resultantes al final de la síntesis fueron analizadas por Espectroscopía Infrarroja (FT-IR). En el caso de las dispersiones resultantes, se recurrió también a una caracterización por Dispersión Dinámica de Luz (DLS) para determinar las distribuciones de tamaño medio de partícula.

El análisis de FT-IR fue hecho en un equipo Thermo Nicolet Nexus 670 FT-IR en modo de transmisión con una resolución $\sim 4 \mathrm{~cm}^{-1}$ en el intervalo $400-4000 \mathrm{~cm}^{-1}$. La fuente de excitación fue la luz de un láser $\mathrm{He}-\mathrm{Ne}$ incidente en un blanco compacto de $\mathrm{KBr}$ que contenía 3 $\mu \mathrm{l}$ de la solución bajo estudio. Para las soluciones de CIS:Hex finales, el estudio fue hecho 10 minutos después para permitir la evaporación del Hexano.

Para el estudio del tamaño medio de partícula por DLS, se dispensaron aproximadamente $1.5 \mathrm{ml}$ de cada muestra de interés en celdas de vidrio tipo PCS1115 que

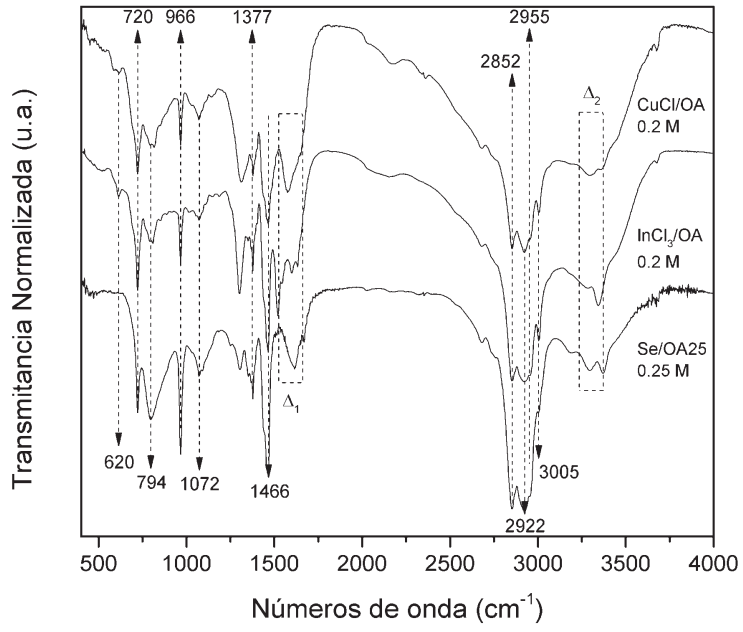

Figura 1: Espectros vibracionales FT-IR de las soluciones precursoras de $\mathrm{Cu}$, In y $\mathrm{Se}$ en $\mathrm{OA}$ según se describió en la sección "Parte Experimental".

eran introducidas en un Zetasizer Nano ZS90. Este equipo cuenta con un láser de $\operatorname{HeNe}(\lambda=633 \mathrm{~nm}) \mathrm{y}$, como detector, un fotodiodo de avalancha con eficiencia cuántica mayor que el $50 \%$ para la longitud de onda de trabajo. Fue necesario también proporcionarle al software del fabricante algunas características del medio dispersante (Hex) necesarias para el posterior procesamiento de la luz dispersada y la estimación del tamaño de partícula. Estos parámetros fueron viscosidad $(\sim 0.3100 \mathrm{cP})$ e índice de refracción $(\sim 1.375)$.

\section{Resultados y discusión}

\subsection{Estudio FT-IR de las soluciones iniciales}

La Figura 1 muestra los espectros vibracionales FT-IR de las soluciones de partida con algunos modos particularmente destacados y asignados según se especifica en la Tabla 1. Como se ve, predominan los modos asociados a los grupos característicos de la Oleilamina: grupos -oleil entre 2850 y $3000 \mathrm{~cm}^{-1}$ y amina en 1593 y $3300 \mathrm{~cm}^{-1}$ aproximadamente. Sin embargo, llama la atención el modo vibracional a 620 $\mathrm{cm}^{-1}$ que aparece para las soluciones precursoras de $\mathrm{CuCl} / \mathrm{OA}$ e InCl3/OA. Dicho modo corresponde a vibraciones $v(\mathrm{C}-\mathrm{Cl})$ e indica que, durante la síntesis de los precursores, se produce la cloración de algunos o varios de los grupos metil (-CH3), metileno (-CH2) o metino $(=\mathrm{CH})$ pertenecientes a la molécula de de Oleilamina. En el caso del precursor Se/OA25, la única síntesis que no involucra cloruros, dicho modo no se observa. Un espectro de características similares fue obtenido para el precursor Se/OA40 y, por tanto, no se muestra.

Por otra parte, el pequeño corrimiento al rojo mostrado por algunos modos vibracionales, especialmente por los asociados a las coordinaciones - $\mathrm{NH}$ y $-\mathrm{NH} 2$, puede estar vinculado a la propiedad de la Oleilamina como agente de terminación de cadena, 'capping agent', una propiedad suficientemente bien documentada y explotada en la síntesis de nanopartículas metálicas. 
Tabla 1: Algunos modos vibracionales observados en los espectros mostrados en la Figura 1.

\begin{tabular}{|c|c|c|}
\hline $\begin{array}{l}\text { Número de } \\
\text { Onda }\left(\mathrm{cm}^{-1}\right)\end{array}$ & $\begin{array}{c}\text { Modos } \\
\text { Vibracionales }\end{array}$ & Referencia \\
\hline 620 & $v(\mathrm{C}-\mathrm{Cl})$ & $830-560 \mathrm{~cm}^{-1} 1718$ \\
\hline 720 & $\delta\left(\mathrm{CH}_{2}\right)$ & $720 \mathrm{~cm}^{-1} 1718$ \\
\hline 794 & $\delta\left(\mathrm{NH}_{2}\right)$ & $787 \mathrm{~cm}^{-1} 171920$ \\
\hline 966 & $\operatorname{trans} \delta(-\mathrm{CH}=)$ & $970 \mathrm{~cm}^{-1} 19$ \\
\hline 1072 & $\delta(=\mathrm{CH}-)$ & $1066 \mathrm{~cm}^{-1} 2122$ \\
\hline 1377 & $\delta_{\mathrm{s}}\left(\mathrm{CH}_{3}\right)$ & $1380 \mathrm{~cm}^{-1} 17$ \\
\hline 1466 & $\delta_{\mathrm{s}}\left(\mathrm{CH}_{2}\right)$ & $1464 \mathrm{~cm}^{-1} 20$ \\
\hline Intervalo $\Delta_{1}$ & $v\left(\mathrm{NH}_{2}\right), v(-\mathrm{C}=\mathrm{C})$ & $23-26$ \\
\hline 2852 & $v_{\mathrm{s}}\left(-\mathrm{CH}_{2}\right)$ & $2854 \mathrm{~cm}^{-1} 19$ \\
\hline 2922 & $v_{\mathrm{a}}\left(-\mathrm{CH}_{2}\right)$ & $2922 \mathrm{~cm}^{-1} 19$ \\
\hline 2955 & $v_{\mathrm{a}}\left(-\mathrm{CH}_{3}\right)$ & $2954 \mathrm{~cm}^{-1} 20$ \\
\hline 3005 & Cis $v(-\mathrm{CH}=)$ & $3006 \mathrm{~cm}^{-1} 19$ \\
\hline Intervalo $\Delta_{2}$ & $\begin{array}{c}\text { Modos asociados } \\
\text { a } v(\mathrm{NH})\end{array}$ & $23-26$ \\
\hline
\end{tabular}

\subsection{Estudio FT-IR de las soluciones finales}

La Figura 2 muestra los espectros FTIR del material sintetizado dispersado en Hexano a diversas relaciones volumétricas. En dicha figura, así como en la Tabla 2, se muestran los mismos modos que fueron destacados y asignados con anterioridad en la Figura / Tabla 1. Como se aprecia, sigue predominando la presencia de modos asociados a la grupos característicos de la Oleilamina aunque esta vez el corrimiento al rojo de algunos modos vibracionales es mucho más notable, en especial los modos asociados a vibraciones $-\mathrm{NH}_{2}$ y $-\mathrm{NH}$. Este comportamiento reafirma el rol de la Oleilamina como agente de terminación de cadena (estabilizador) además de ser, a la vez, solvente.

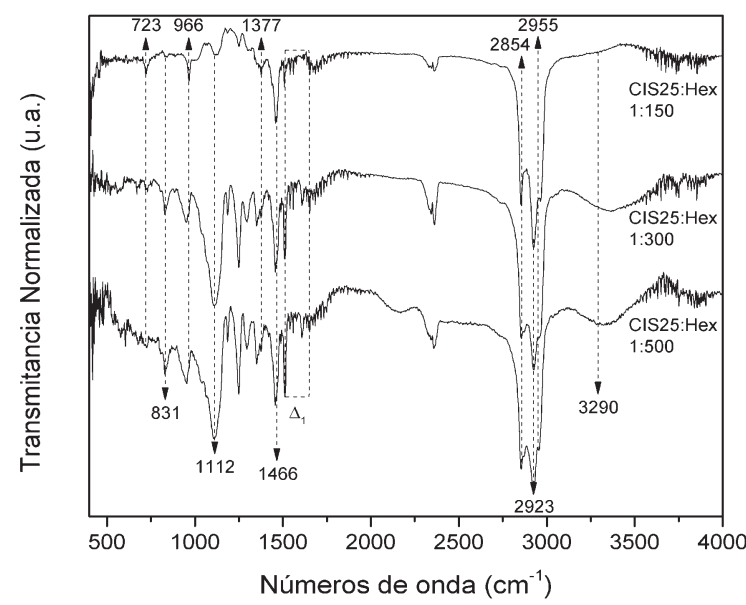

Figura 2: Espectros vibracionales FT-IR de las soluciones de CIS:Hex analizadas.
Por otra parte, el modo $\mathrm{C}-\mathrm{Cl}$, asociado anteriormente a la probable cloración de grupos metil $\left(-\mathrm{CH}_{3}\right)$, metileno ($\mathrm{CH}_{2}$ ) o metino $(=\mathrm{CH})$, no fue distinguible esta vez lo cual nos hace valorar que, para la temperatura de síntesis de $250{ }^{\circ} \mathrm{C}$, es bastante plausible que ocurra alguna evaporación de $\mathrm{HCl}(\mathrm{g})$ así como también una reacción entre el $\mathrm{HCl}$ con el exceso de Oleilamina formando probablemente una sal hidroclórica de la amina. Como en la discusión anterior, debe decirse que un espectro de características similares fue obtenido para el material CIS40:Hex (sintetizado con el precursor Se/OA40) y, por tanto, tampoco se muestra.

Llegados a este punto, podríamos escribir las ecuaciones químicas más esperadas correspondientes a los escenarios antes discutidos. Así, podría suponerse:

$\mathrm{CuCl}+\mathrm{InCl}_{3}+2 \mathrm{Se}+8 \mathrm{C}_{17} \mathrm{H}_{33}-\mathrm{CH}_{2}-\mathrm{NH}_{2}=\left[\mathrm{CuInSe}_{2}+\right.$ $\left.6 \mathrm{C}_{17} \mathrm{H}_{33}-\mathrm{CH}_{2}-\mathrm{NH}_{2}\right]+x \mathrm{HCl}+(2-\mathrm{x}) \mathrm{C}_{17} \mathrm{H}_{33}-\mathrm{CH}_{2}-\mathrm{NH}_{3}{ }^{+} \mathrm{Cl}^{-}$ [a $250{ }^{\circ} \mathrm{C} / 2 \mathrm{hrs}$,

donde hemos asumido, hipotéticamente, la estabilización de cada molécula de $\mathrm{CuInSe}_{2}$ por 6 moléculas de OA $\left[\mathrm{OA}=\mathrm{C}_{17} \mathrm{H}_{33}-\mathrm{CH}_{2}-\mathrm{NH}_{2}\right]$, la evaporación de una fracción $\mathrm{x}$ de $\mathrm{HCl}$ y la formación de una fracción (2-x) de la sal hidroclórica de la amina. En realidad, como la reacción ocurre con exceso de $\mathrm{OA}$, debería esperarse a existencia paralela de todos estos fenómenos.

Tabla 2: Algunos modos vibracionales observados en los espectros mostrados en la Figura 2. Para una mejor apreciación del corrimiento, se vuelven a mostrar los analizados antes en la Figura 1 y en la Tabla 1.

\begin{tabular}{ccc}
\hline $\begin{array}{c}\text { Número de } \\
\text { Onda }\left(\mathbf{c m}^{-1}\right)\end{array}$ & $\begin{array}{c}\text { Modos } \\
\text { Vibracionales }\end{array}$ & Referencia \\
\hline 723 & $\delta\left(\mathrm{CH}_{2}\right)$ & $720 \mathrm{~cm}^{-1} 1718$ \\
831 & $\delta\left(\mathrm{NH}_{2}\right)$ & $787 \mathrm{~cm}^{-1}$ \\
966 & trans $\delta(-\mathrm{CH}=)$ & $970 \mathrm{~cm}^{-1} 19$ \\
1112 & $\delta(=\mathrm{CH}-)$ & $1066 \mathrm{~cm}^{-1} 2122$ \\
1377 & $\delta_{\mathrm{s}}\left(\mathrm{CH}_{3}\right)$ & $1380 \mathrm{~cm}^{-1} 17$ \\
1466 & $\delta_{\mathrm{s}}\left(\mathrm{CH}_{2}\right)$ & $1464 \mathrm{~cm}^{-1} 20$ \\
Intervalo $\Delta_{1}$ & $v\left(\mathrm{NH}_{2}\right), v(-\mathrm{C}=\mathrm{C})$ & $23-26$ \\
2854 & $v_{\mathrm{s}}\left(-\mathrm{CH}_{2}\right)$ & $2854 \mathrm{~cm}^{-1} 19$ \\
2923 & $v_{\mathrm{a}}\left(-\mathrm{CH}_{2}\right)$ & $2922 \mathrm{~cm}^{-1} 19$ \\
2955 & $v_{\mathrm{a}}\left(-\mathrm{CH}_{3}\right)$ & $2954 \mathrm{~cm}^{-1} 20$ \\
3290 & $v(\mathrm{NH})$ & $23-26$ \\
\hline
\end{tabular}




\subsection{Estudio del tamaño de partícula en las soluciones finales}

Como ya se dijo, el material final obtenido fue dispersado en Hexano a distintas razones volumétricas y caracterizado por DLS para analizar el tamaño medio de partícula. La Figura 3 muestra dichos resultados.

Primeramente salta a la vista que el tamaño medio de partícula rebasa los $1000 \mathrm{~nm}(1 \mu \mathrm{m})$ de diámetro hidrodinámico lo cual puede verse vinculado a las propiedades del hexano (solvente apolar) como "floculador" o "agente floculante". Nótese también, de paso, cómo aumenta el tamaño medio de partícula con la concentración de Hexano. En otras palabras: la medición se está realizando sobre un sistema formado por arreglos micrométricos de nanopartículas de $\mathrm{CuInSe}_{2}$.

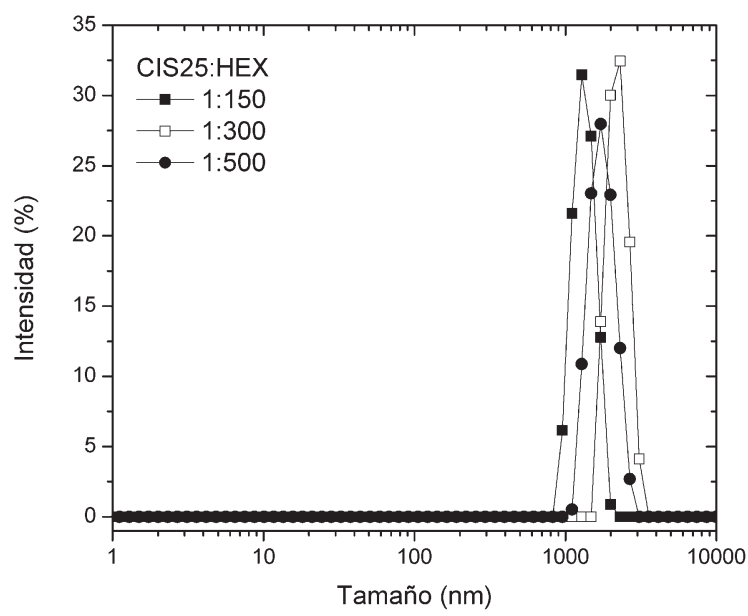

Figura 3: Análisis por DLS de tamaño medio de partícula para las soluciones CIS25:Hex con diferentes razones volumétricas.

Esta característica, a primera vista, no parece ser despreciable pensando en las aplicaciones prácticas en forma de "tintura semiconductora" pues garantizaría una mejor continuidad de la capa activa depositada por el método de impresión que se elija. Sin embargo, lo que

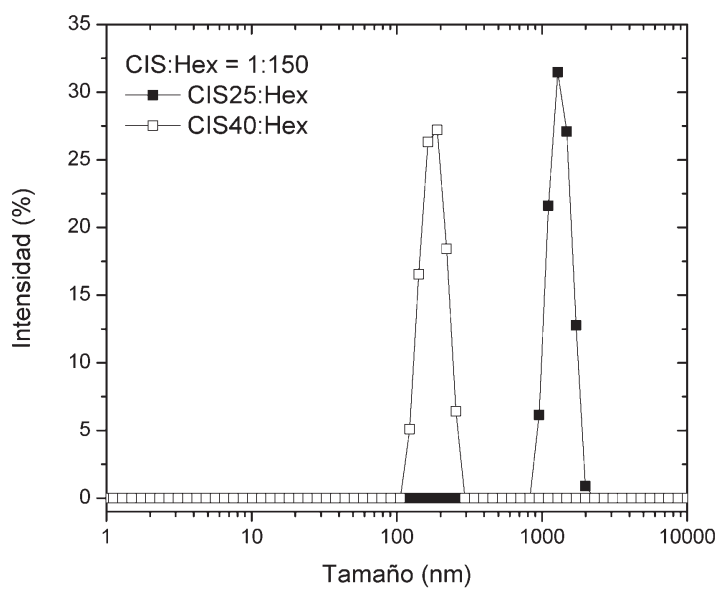

Figura 4: Análisis por DLS de tamaño medio de partícula para las soluciones CIS25:Hex y CIS40:Hex con idénticas razones volumétricas 1:500. deja de hacerla apropiada para fines prácticos es precisamente el elevado nivel de dilución (hasta 1:500 en este estudio) que se requiere.

Por otra parte, las soluciones CIS40:Hex muestran unas distribuciones de tamaño de partícula mucho más pequeño que las CIS25:Hex. La Figura 4 muestra comparativamente los resultados arrojados por DLS para los especímenes CIS25 y CIS40 sintetizados y tratados idénticamente en el experimento además de diluidos en Hexano bajo las mismas condiciones y la misma relación volumétrica.

Como se ve, la solución basada en CIS40 presenta un tamaño medio de partícula casi 10 veces menor a la CIS25. Tal disminución podría estar dada, entre otros factores, por la menor cantidad de Oleilamina involucrada en la reacción (un $37.5 \%$ menos) lo cual implica una menor razón de moléculas estabilizadoras por cada molécula de $\mathrm{CuInSe}_{2} \mathrm{y}$, a su vez, un menor apantallamiento de las cargas efectivas (repulsión coulombiana) evitando la fácil floculación y permitiendo, a su vez, la existencia de aglomerados más pequeños en presencia de algún agente floculante como el Hexano.

Pese a lo dicho, otras explicaciones podrían ser exploradas. Una de ellas debe estar relacionada con el cuadro físico-químico asociado a la incorporación del elemento $\mathrm{Se}$ en el momento de la síntesis pues es conocida la formación de compuestos intermedios del Selenio con $\mathrm{Cu}$ e In de relativa estabilidad y, como es de esperarse, el orden de adición de los reactivos es, en general, un factor nfluyente en las características del material final.

Ambos escenarios, el relativo a la correlación de fuerzas atractivas-repulsivas en el seno de una dispersión coloidal así como el relativo a los mecanismos de incorporación del Selenio (Selenización), constituyen objetos de estudio para futuros trabajos.

\section{Conclusiones}

En el trabajo descrito se formuló, basado puramente en análisis FT-IR, un probable esquema de reacción para la formación de nanopartículas de $\mathrm{CuInSe}_{2}$ en nuestros experimentos. Fue analizado también el tamaño medio de partícula por DLS para soluciones sintetizadas bajo diferentes condiciones de Selenización y se verificó la influencia de la misma en las características del material final. Las tinturas semiconductoras de $\mathrm{CuInSe}_{2}$ sintetizadas en este trabajo mostraron viscosidades apropiadas para el depósito, mediante pintura convencional, sobre un substrato dado. Pasadas 6 semanas después de la síntesis, dichas tinturas no mostraron señales notables de envejecimiento en lo que a tamaño medio de partícula se refiere.

Tomando en cuenta que un estudio basado únicamente en FT-IR no es conclusivo en el análisis estructural del material aquí tratado, un paso posterior a este trabajo deberá ser la determinación precisa de la calidad estructural y morfológica del material final obtenido mediante el uso de las técnicas apropiadas: XRD, Raman, SEM y TEM, entre otras. Dichos estudios formarán parte de un próximo trabajo, aun en desarrollo. 


\section{Agradecimientos}

Se reconoce el apoyo brindado por COECYTJAL (Proy. No. 5-2010-1-937) para la realización de este trabajo.

\section{Referencias}

1. Ward, J. S.; Ramanathan, K.; Hasoon, F. S.; Coutts, T. J.; Keane, J.; Contreras, M. A.; Moriarty, T.; Noufi, R. Prog. Photovoltaics 2002, 10 (1), 41-46.

2. Little, R. G.; Nowlan, M. J. Prog. Photovoltaics 1997, 5 (5), 309-315

3. Ullal, H. S.; Zweibel, K.; von Roedern, B. G. Photovoltaic Specialists Conference, 2000. Conference Record of the Twenty-Eighth IEEE, 2000; 2000; pp 418-423.

4. Guillemoles, J.-F.; Rau, U.; Kronik, L.; Schock, H.-W.; Cahen, D. Adv. Mater. 1999, 11 (11), 957-961.

5. Stanbery, B. J. Crit. Rev. Solid State Mater. Sci. 2002, 27 (2), 73-117.

6. Ramanathan, K.; Contreras, M. A.; Perkins, C. L.; Asher, S.; Hasoon, F. S.; Keane, J.; Young, D.; Romero, M.; Metzger, W.; Noufi, R.; Ward, J.; Duda, A. Prog. Photovoltaics 2003, 11 (4), 225-230.

7. Joo, J.; Na, H. B.; Yu, T.; Yu, J. H.; Kim, Y. W.; Wu, F.; Zhang, J. Z.; Hyeon, T. J. Am. Chem. Soc. 2003, $125(36), 11100-5$.

8. Trindade, T.; O'Brien, P.; Pickett, N. L. Chem. Mater. 2001, 13 (11), 3843-3858.

9. Murray, C. B.; Kagan, C. R.; Bawendi, M. G. Annu. Rev. Mater. Sci. 2000, 30 (1), 545-610.

10. Kaelin, M.; Rudmann, D.; Kurdesau, F.; Meyer, T.; Zogg, H.; Tiwari, A. N. Thin Solid Films 2003, 431432, 58-62.

11. Smyder, J. A.; Krauss, T. D. Mater. Today 2011, 14 (9), 382-387.

12. Shen, F.; Que, W.; Zhong, P.; Zhang, J.; Yin, X. Colloids Surf., A 2011, 392 (1), 1-6.

13. Guo, Q.; Kim, S. J.; Kar, M.; Shafarman, W. N.; Birkmire, R. W.; Stach, E. A.; Agrawal, R.; Hillhouse, H. W. Nano Lett. 2008, 8 (9), 2982-7.

14. Zou, Y.; Li, D.; Yang, D. Mater. Chem. Phys. 2012, $132(2-3), 865-869$.

15. Zhang, J.; Que, W.; Shen, F.; Liao, Y. Sol. Energy Mater. Sol. Cells 2012, 103, 30-34.

16. Wang, W.-J.; Jiang, Y.; Lan, X.-Z.; Wang, C.; Liu, X.M.; Wang, B.-B.; Li, J.-W.; Yang, B.; Ding, X.-N. Mater. Sci. Semicond. Process. 2012, 15 (5), 467-471.

17. Kar, M.; Agrawal, R.; Hillhouse, H. W. J. Am. Chem. Soc. 2011, 133 (43), 17239-47.

18. Günzler, H.; Gremlich, H.U. IR Spectroscopy. An introduction; Weinheim : Wiley-VCH, 2002.

19. Shukla, N.; Svedberg, E. B.; Ell, Colloids Surf., A 2007, 301 (1-3), 113-116.

20. Klokkenburg, M.; Hilhorst, J.; Erné, B. H. Vib. Spectrosc. 2007, 43 (1), 243-248.

21. Chen, M.; Feng, Y. G.; Wang, X.; Li, T. C.; Zhang, J. Y.; Qian, D. J. Langmuir 2007, 23 (10), 5296-304.

22. Polavarapu, L.; Venkatram, N.; Ji, W.; Xu, Q. H. ACS Appl. Mater. Interfaces 2009, 1 (10), 2298-303.

23. Shukla, N.; Liu, C.; Jones, P. M.; Weller, D. J. Magn. Magn. Mater. 2003, 266 (1-2), 178-184.

24. Polavarapu, L.; Xu, Q. H. Nanotechnology 2009, 20 (18), 185606.

25. Tzitzios, V.; Georgakilas, V.; Oikonomou, E.; Karakassides, M.; Petridis, D. Carbon 2006, 44 (5), 848-853.

26. Feng, H.; Yang, Y.; You, Y.; Li, G.; Guo, J.; Yu, T.; Shen, Z.; Wu, T.; Xing, B. Chem. Commun. 2009, (15), 1984-6. 